\title{
STARLIKENESS ASSOCIATED WITH PARABOLIC REGIONS
}

\author{
ROSIHAN M. ALI
}

Received 29 April 2004 and in revised form 15 September 2004

A parabolic starlike function $f$ of order $\rho$ in the unit disk is characterized by the fact that the quantity $z f^{\prime}(z) / f(z)$ lies in a given parabolic region in the right half-plane. Denote the class of such functions by $\operatorname{PS}^{*}(\rho)$. This class is contained in the larger class of starlike functions of order $\rho$. Subordination results for $\operatorname{PS}^{*}(\rho)$ are established, which yield sharp growth, covering, and distortion theorems. Sharp bounds for the first four coefficients are also obtained. There exist different extremal functions for these coefficient problems. Additionally, we obtain a sharp estimate for the Fekete-Szegö coefficient functional and investigate convolution properties for $\operatorname{PS}^{*}(\rho)$.

\section{Introduction}

Let $A$ denote the class of analytic functions $f$ in the open unit disk $U=\{z:|z|<1\}$ and let $f$ be normalized so that $f(0)=f^{\prime}(0)-1=0$. In [4], Goodman introduced the class UCV of uniformly convex functions consisting of convex functions $f \in A$ with the property that for every circular arc $\gamma$ contained in $U$, with center also in $U$, the image arc $f(\gamma)$ is a convex arc. He derived a two-variable characterization of functions in UCV, that is, $f \in A$ belongs to UCV if and only if for every pair $(z, \varsigma) \in U \times U$,

$$
1+\mathfrak{R}\left\{(z-\varsigma) \frac{f^{\prime \prime}(z)}{f^{\prime}(z)}\right\} \geq 0 .
$$

Ma and Minda [6] and Rønning [10] independently developed a one-variable characterization that $f \in \mathrm{UCV}$ if and only if for every $z \in U$,

$$
\left|\frac{z f^{\prime \prime}(z)}{f^{\prime}(z)}\right|<\mathfrak{R}\left(1+\frac{z f^{\prime \prime}(z)}{f^{\prime}(z)}\right) .
$$

Rønning [10] also showed that $f \in \mathrm{UCV}$ if and only if the function $z f^{\prime} \in \mathrm{PS}^{*}$, where PS* is the class of functions $g \in A$ satisfying

$$
\left|\frac{z g^{\prime}(z)}{g(z)}-1\right|<\mathfrak{R} \frac{z g^{\prime}(z)}{g(z)}, \quad z \in U .
$$


Several authors have studied the classes above, amongst which the authors of $[4,6,7,8$, $9,10,12]$.

In [9], the class PS* was generalized by looking at functions $f \in A$ satisfying

$$
\left|\frac{z f^{\prime}(z)}{f(z)}-1\right|<\mathfrak{R} \frac{z f^{\prime}(z)}{f(z)}-\alpha, \quad z \in U .
$$

In this paper, we continue the investigation of this generalized class but under a slight modification of parameter. For $0 \leq \rho<1$, let $\Omega_{\rho}$ be the parabolic region in the right halfplane

$$
\Omega_{\rho}=\left\{w=u+i v: v^{2}<4(1-\rho)(u-\rho)\right\}=\{w:|w-1|<1-2 \rho+\Re w\} .
$$

The class of parabolic starlike functions of order $\rho$ is the subclass PS $(\rho)$ of $A$ consisting of functions $f$ such that $z f^{\prime}(z) / f(z) \in \Omega_{\rho}, z \in U$. Thus $f \in \operatorname{PS}^{*}(\rho)$ if and only if for $z \in U$,

$$
\left|\frac{z f^{\prime}(z)}{f(z)}-1\right|<1-2 \rho+\mathfrak{R} \frac{z f^{\prime}(z)}{f(z)} .
$$

Similarly, a function $f \in A$ belongs to $\operatorname{UCV}(\rho)$ if and only if for every pair $(z, \varsigma)$ in the polydisk $U \times U$,

$$
1+\mathfrak{R}\left\{(z-\varsigma) \frac{f^{\prime \prime}(z)}{f^{\prime}(z)}\right\}>2 \rho-1
$$

A function $f \in \operatorname{UCV}(\rho)$ is called an uniformly convex function of order $\rho$. Thus the classes discussed earlier correspond to UCV $=\mathrm{UCV}(1 / 2)$ and PS $*=\mathrm{PS}^{*}(1 / 2)$. In [5], Lee showed that

$$
g \in \mathrm{UCV}(\rho) \Longleftrightarrow f=z g^{\prime} \in \operatorname{PS}^{*}(\rho)
$$

that is,

$$
g \in \mathrm{UCV}(\rho) \Longleftrightarrow\left|\frac{z g^{\prime \prime}(z)}{g^{\prime}(z)}\right|<2(1-\rho)+\mathfrak{R} \frac{z g^{\prime \prime}(z)}{g^{\prime}(z)}
$$

In the present paper, we continue the study of $\operatorname{PS}^{*}(\rho)$ realized by Ali and Singh [3], and more recently by Aghalary and Kulkarni [1]. We give examples of functions in the class PS* $\left.^{*} \rho\right)$, and establish subordination results, which yield sharp growth, covering and distortion theorems. Sharp bounds on the first four coefficients are also obtained. There exist different extremal functions for these coefficient problems. Additionally, we obtain a sharp estimate for the Fekete-Szegö coefficient functional and examine convolution properties for $\operatorname{PS}^{*}(\rho)$.

\section{Preliminary results}

From its definition, it is clear that the class $\operatorname{PS}^{*}(\rho)$ is contained in the class $S^{*}(\rho)$ of starlike functions of order $\rho$, that is, $\mathfrak{R}\left(z f^{\prime}(z) / f(z)\right)>\rho, z \in U$. It is also fairly immediate 
that $\operatorname{PS}^{*}(\rho)$ is related to the class of strongly starlike functions, where a function $f \in A$ is said to be strongly starlike of order $\alpha, 0<\alpha \leq 1$, if $f$ satisfies $\left|\operatorname{Arg} z f^{\prime}(z) / f(z)\right|<\pi \alpha / 2$, $z \in U$. We state the relation in the theorem below.

THEOREM 2.1. If $f \in \operatorname{PS}^{*}(\rho)$, then $f$ is strongly starlike of order $\gamma$, where $(\pi / 2) \gamma=$ $\tan ^{-1} \sqrt{(1-\rho) / \rho}$. In other words, for $z \in U$,

$$
\left|\operatorname{Arg} \frac{z f^{\prime}(z)}{f(z)}\right| \leq \frac{\pi \gamma}{2}
$$

A sufficient condition for a function $f$ to be parabolic starlike of order $\rho$ is given by the following theorem.

Theorem 2.2. If $f \in A$ satisfies

$$
\left|\frac{z f^{\prime}(z)}{f(z)}-1\right|<1-\rho
$$

then $f \in \operatorname{PS}^{*}(\rho)$.

Proof. The given condition implies that

$$
\mathfrak{R} \frac{z f^{\prime}(z)}{f(z)}-\left|\frac{z f^{\prime}(z)}{f(z)}-1\right|+1-2 \rho \geq 2(1-\rho)-2\left|\frac{z f^{\prime}(z)}{f(z)}-1\right|>0 .
$$

The following two examples are now easily established from Theorem 2.2.

Example 2.3. The function $f(z)=z+\alpha z^{n} \in \mathrm{PS}^{*}(\rho)$ if and only if $|\alpha| \leq(1-\rho) /(n-\rho)$.

Example 2.4. The generalized hypergeometric function is defined by

$$
F\left(a_{1}, \ldots, a_{p} ; b_{1}, \ldots, b_{q} ; z\right)=1+\sum_{n=1}^{\infty} \frac{\left(a_{1}\right)_{n} \cdots\left(a_{p}\right)_{n}}{\left(b_{1}\right)_{n} \cdots\left(b_{q}\right)_{n}} \frac{z^{n}}{n !}, \quad b_{j} \neq 0,-1, \ldots,
$$

where $(\lambda)_{n}$ is the Pochhammer symbol defined by

$$
(\lambda)_{n}= \begin{cases}1, & n=0, \\ \lambda(\lambda+1)(\lambda+2) \cdots(\lambda+n-1), & n=1,2, \ldots\end{cases}
$$

If $\left|z F^{\prime}(z) / F(z)\right|<1-\rho$, then $z F \in \operatorname{PS}^{*}(\rho)$.

Ali and Singh [3] showed that the normalized Riemann mapping function $q_{\rho}$ from $U$ onto $\Omega_{\rho}$ is given by

$$
q_{\rho}(z)=1+\frac{4(1-\rho)}{\pi^{2}}\left[\log \frac{1+\sqrt{z}}{1-\sqrt{z}}\right]^{2}=1+\sum_{n=1}^{\infty} B_{n} z^{n}
$$


Here

$$
B_{1}=\frac{16(1-\rho)}{\pi^{2}}, \quad B_{n}=\frac{16(1-\rho)}{n \pi^{2}} \sum_{k=0}^{n-1} \frac{1}{2 k+1}, \quad n=2,3, \ldots
$$

Since the latter sum is bounded above by $1+(1 / 2) \log (2 n-1)$ (see [6]) an upper bound for each coefficient is given by

$$
B_{n}<\frac{16(1-\rho)}{n \pi^{2}}\left(1+\frac{1}{2} \log (2 n-1)\right) .
$$

However these bounds do not yield sharp coefficient estimates for the class $\operatorname{PS}^{*}(\rho)$. We will return to the coefficient problem in the next section.

Let $k \in \mathrm{PS}^{*}(\rho)$ be defined by $k(0)=k^{\prime}(0)-1=0$ and

$$
\frac{z k^{\prime}(z)}{k(z)}=q_{\rho}(z)
$$

In [8], Ma and Minda established a general result that leads to the following result.

Theorem 2.5 [8]. If $f \in \operatorname{PS}^{*}(\rho)$, then

(a) $z f^{\prime}(z) / f(z) \prec z k^{\prime}(z) / k(z)$ and $f(z) / z \prec k(z) / z$,

(b) $-k(-r) \leq|f(z)| \leq k(r),|z| \leq r<1$,

(c) $|\operatorname{Arg}(f(z) / z)| \leq \max _{|z|=r}|\operatorname{Arg}(k(z) / z)|,|z| \leq r<1$,

(d) $k^{\prime}(-r) \leq\left|f^{\prime}(z)\right| \leq k^{\prime}(r),|z| \leq r<1$.

Equality in (b), (c), and (d) holds for some $z \neq 0$ if and only if $f$ is a rotation of $k$.

Since the function $k$ is continuous in $\bar{U},-k(-1)=\lim _{r \rightarrow 1}-k(-r)$ and $k(1)=$ $\lim _{r \rightarrow 1} k(r)$ exist. Rønning [9] established the following corollary.

Corollary 2.6 [9]. (a) Let $f \in \operatorname{PS}^{*}(\rho)$. Then either $f$ is a rotation of $k$ or $f(U) \supset\{w$ : $|w| \leq-k(-1)\}$, where the Koebe constant is $-k(-1)=e^{-(1-\rho)(1.25475)}$.

(b) The functions in $\operatorname{PS}^{*}(\rho)$ are uniformly bounded by the sharp constant $k(1)=$ $e^{3.41023(1-\rho)}$.

\section{Coefficient bounds}

We first give another sufficient condition for a function $f$ to belong to $\operatorname{PS}^{*}(\rho)$.

THeOrem 3.1. If $f(z)=z+\sum_{n=2}^{\infty} a_{n} z^{n}$ satisfies $\sum_{n=2}^{\infty}(n-1)\left|a_{n}\right| \leq(1-\rho) /(2-\rho)$, then $f \in \operatorname{PS}^{*}(\rho)$. The constant $(1-\rho) /(2-\rho)$ cannot be replaced by a larger number.

Proof. Let $g(z)=\int_{0}^{z}(f(\xi) / \xi) d \xi=z+\sum_{n=2}^{\infty}\left(a_{n} / n\right) z^{n}$. In view of (1.8), it suffices to show that $g \in \operatorname{UCV}(\rho)$. Since

$$
\sum_{n=2}^{\infty}\left|a_{n}\right| \leq \frac{1-\rho}{2-\rho}
$$


it follows that

$$
1+\mathfrak{R}(z-\varsigma) \frac{g^{\prime \prime}(z)}{g^{\prime}(z)} \geq 1-\frac{\sum_{n=2}^{\infty}(n-1)\left|a_{n}\right||z|^{n-2}}{1-\sum_{n=2}^{\infty}\left|a_{n}\right||z|^{n-1}}|z-\varsigma| \geq 2 \rho-1 .
$$

Thus $g \in \operatorname{UCV}(\rho)$. The function $f(z)=z+((1-\rho) /(2-\rho)) z^{2}$ in Example 2.3 shows that the constant $(1-\rho) /(2-\rho)$ is the best possible.

We next consider the problem of finding

$$
A_{n}=\max _{f \in \mathrm{PS}^{*}(\rho)}\left|a_{n}\right|
$$

If $f(z)=z+a_{2} z^{2}+a_{3} z^{3}+\cdots \in$ PS $^{*}(\rho)$ and $h(z)=z f^{\prime}(z) / f(z)$, then there exists a Schwarz function $w$ defined in $U$ with $w(0)=0,|w(z)|<1$, and satisfying

$$
h(z)=\frac{z f^{\prime}(z)}{f(z)}=q_{\rho}(w(z))
$$

If $h(z)=1+b_{1} z+b_{2} z^{2}+\cdots$, the first equality in (3.4) implies that

$$
(n-1) a_{n}=\sum_{k=1}^{n-1} a_{k} b_{n-k}
$$

Since $q_{\rho}$ is univalent in $U$ and $h \prec q_{\rho}$, the function

$$
p(z)=\frac{1+q_{\rho}^{-1}(h(z))}{1-q_{\rho}^{-1}(h(z))}=1+c_{1} z+c_{2} z^{2}+\cdots
$$

belongs to the class $P$ consisting of analytic functions $p$ in the unit disk $U$ with positive real part such that $p(0)=1$ and $\Re p(z)>0, z \in U$. In other words,

$$
h(z)=q_{\rho}\left(\frac{p(z)-1}{p(z)+1}\right) .
$$

While (3.5) gives $a_{n}$ in terms of the coefficients $b_{k}$, (3.7) expresses the $b_{k}$ 's in terms of the coefficients $c_{m}$ 's and $B_{m}$ 's. It is now easily established that

$$
\begin{aligned}
& a_{2}=\frac{8(1-\rho)}{\pi^{2}} c_{1}, \\
& a_{3}=\frac{8(1-\rho)}{2 \pi^{2}}\left[c_{2}-\left(\frac{1}{6}-\frac{8(1-\rho)}{\pi^{2}}\right) c_{1}^{2}\right], \\
& a_{4}=\frac{8(1-\rho)}{3 \pi^{2}}\left[c_{3}-\left(\frac{1}{3}-\frac{12(1-\rho)}{\pi^{2}}\right) c_{1} c_{2}+\left(\frac{2}{45}-\frac{2(1-\rho)}{\pi^{2}}+\frac{32(1-\rho)^{2}}{\pi^{4}}\right) c_{1}^{3}\right] .
\end{aligned}
$$

Thus the coefficient estimates for PS* $(\rho)$ may be viewed in terms of nonlinear coefficient problems for the class $P$. 

by

We now introduce the following functions in $\operatorname{PS}^{*}(\rho)$. Define $k_{n}, G, H \in A$, respectively,

$$
\frac{z k_{n}^{\prime}(z)}{k_{n}(z)}=q_{\rho}\left(z^{n-1}\right), \quad \frac{z H^{\prime}(z)}{H(z)}=q_{\rho}\left(\frac{z(z-r)}{1-r z}\right), \quad \frac{z G^{\prime}(z)}{G(z)}=q_{\rho}\left(-\frac{z(z-r)}{1-r z}\right), \quad 0 \leq r \leq 1 .
$$

It is clear from (3.4) that $k_{n}, G, H \in \mathrm{PS}^{*}(\rho)$, and that $k_{2}(z)=k(z)$. Since

$$
k_{n}(z)=z+\frac{16(1-\rho)}{(n-1) \pi^{2}} z^{n}+\cdots,
$$

we find that

$$
A_{n} \geq \frac{16(1-\rho)}{(n-1) \pi^{2}}
$$

On the other hand, Ali and Singh [3] proved that

$$
(n-1) A_{n} \leq 2 \sqrt{2}(1-\rho) e^{4(1-\rho)^{2}},
$$

which also yields the sharp order of growth $\left|a_{n}\right|=O(1 / n)$.

From a result of Ma and Minda [8], we can also deduce the following solution to the Fekete-Szegö coefficient functional over the class PS* $(\rho)$. We will omit the details.

Theorem 3.2. Let $f(z)=z+a_{2} z^{2}+a_{3} z^{3}+\cdots \in \operatorname{PS}^{*}(\rho)$. Then

$$
\left|a_{3}-t a_{2}^{2}\right| \leq \begin{cases}\frac{16(1-\rho)}{3 \pi^{4}}\left[24(1-\rho)(1-2 t)+\pi^{2}\right], & t \leq \frac{1}{2}-\frac{\pi^{2}}{96(1-\rho)}, \\ \frac{8(1-\rho)}{\pi^{2}}, & \frac{1}{2}-\frac{\pi^{2}}{96(1-\rho)} \leq t \leq \frac{1}{2}+\frac{5 \pi^{2}}{96(1-\rho)}, \\ \frac{16(1-\rho)}{3 \pi^{4}}\left[24(1-\rho)(2 t-1)-\pi^{2}\right], & t \geq \frac{1}{2}+\frac{5 \pi^{2}}{96(1-\rho)} .\end{cases}
$$

If $1 / 2-\pi^{2} / 96(1-\rho)<t<1 / 2+5 \pi^{2} / 96(1-\rho)$, equality holds if and only if $f=k_{3}$ or one of its rotations. If $t<1 / 2-\pi^{2} / 96(1-\rho)$ or $t>1 / 2+5 \pi^{2} / 96(1-\rho)$, equality holds if and only if $f=k_{2}$ or one of its rotations. If $t=1 / 2-\pi^{2} / 96(1-\rho)$, equality holds if and only if $f=H$ or one of its rotations, while if $t=1 / 2+5 \pi^{2} / 96(1-\rho)$, then equality holds if and only if $f=G$ or one of its rotations.

The above estimates can be used to determine sharp upper bounds on the second and third coefficients, respectively, which we will state below. In addition, the sharp bound on the fourth coefficient $A_{4}$ is determined with the aid of the following lemma.

Lemma 3.3 [2]. Let $p(z)=1+\sum_{k=1}^{\infty} c_{k} z^{k} \in P$. If $0 \leq \beta \leq 1$ and $\beta(2 \beta-1) \leq \delta \leq \beta$, then

$$
\left|c_{3}-2 \beta c_{1} c_{2}+\delta c_{1}^{3}\right| \leq 2 .
$$


In particular,

$$
\left|c_{3}-2 \beta c_{1} c_{2}+\beta c_{1}^{3}\right| \leq 2 \text {. }
$$

When $\beta=0$, equality holds if and only if

$$
p(z):=p_{3}(z)=\sum_{k=1}^{3} \lambda_{k} \frac{1+\epsilon e^{-2 \pi i k / 3} z}{1-\epsilon e^{-2 \pi i k / 3} z}, \quad|\epsilon|=1, \lambda_{k} \geq 0,
$$

with $\lambda_{1}+\lambda_{2}+\lambda_{3}=1$. If $\beta=1$, equality holds if and only if $p$ is the reciprocal of $p_{3}$. If $0<\beta<1$, equality holds if and only if

$$
p(z)=\frac{1+\epsilon z}{1-\epsilon z}, \quad|\epsilon|=1 \quad \text { or } \quad p(z)=\frac{1+\epsilon z^{3}}{1-\epsilon z^{3}}, \quad|\epsilon|=1 .
$$

Theorem 3.4. Let $f(z)=z+a_{2} z^{2}+a_{3} z^{3}+\cdots \in \operatorname{PS}^{*}(\rho)$. Then

$$
\left|a_{2}\right| \leq \frac{16(1-\rho)}{\pi^{2}}
$$

with equality if and only if $f=k$ or its rotations. Further

$$
\left|a_{3}\right| \leq \begin{cases}\frac{8(1-\rho)}{\pi^{2}}\left(\frac{2}{3}+\frac{16(1-\rho)}{\pi^{2}}\right), & 0 \leq \rho \leq 1-\frac{\pi^{2}}{48} \\ \frac{8(1-\rho)}{\pi^{2}}, & 1-\frac{\pi^{2}}{48} \leq \rho<1 .\end{cases}
$$

For $0 \leq \rho<1-\pi^{2} / 48$, equality holds if and only if $f=k$ or its rotations. For $1-\pi^{2} / 48<$ $\rho<1$, equality holds if and only if $f=k_{3}$ or its rotations. If $\rho=1-\pi^{2} / 48$, equality holds if and only if $f=H$ or its rotations. Additionally,

$$
\left|a_{4}\right| \leq \begin{cases}\frac{16(1-\rho)}{3 \pi^{2}}\left[\frac{128(1-\rho)^{2}}{\pi^{4}}+\frac{16(1-\rho)}{\pi^{2}}+\frac{23}{45}\right], & 0 \leq \rho \leq 1+\frac{\pi^{2}}{16}\left(1-\sqrt{\frac{89}{45}}\right), \\ \frac{16(1-\rho)}{3 \pi^{2}}, & 1+\frac{\pi^{2}}{16}\left(1-\sqrt{\frac{89}{45}}\right) \leq \rho<1 .\end{cases}
$$

Equality holds in the upper expression of the right inequality if and only if $f=k$ or its rotations, while equality holds in the lower expression of the right inequality if and only if $f=k_{4}$ or its rotations.

Proof. In the light of Theorem 3.2, we are left to finding an estimate on the fourth coefficient. The relation (3.8) gives

$$
\begin{aligned}
a_{4} & =\frac{8(1-\rho)}{3 \pi^{2}}\left[c_{3}-\left(\frac{1}{3}-\frac{12(1-\rho)}{\pi^{2}}\right) c_{1} c_{2}+\left(\frac{2}{45}-\frac{2(1-\rho)}{\pi^{2}}+\frac{32(1-\rho)^{2}}{\pi^{4}}\right) c_{1}^{3}\right] \\
& :=\frac{8(1-\rho)}{3 \pi^{2}} E .
\end{aligned}
$$


568 Starlikeness associated with parabolic regions

We will apply Lemma 3.3 with

$$
2 \beta=\frac{1}{3}-\frac{12(1-\rho)}{\pi^{2}}, \quad \delta=\frac{2}{45}-\frac{2(1-\rho)}{\pi^{2}}+\frac{32(1-\rho)^{2}}{\pi^{4}} .
$$

The conditions on $\beta$ and $\delta$ are satisfied if

$$
1+\frac{\pi^{2}}{16}\left(1-\sqrt{\frac{89}{45}}\right) \leq \rho<1 .
$$

Thus $\left|a_{4}\right| \leq 16(1-\rho) / 3 \pi^{2}$, with equality if and only if the function $p$ in (3.7) is given by $p(z)=\left(1+\epsilon z^{3}\right) /\left(1-\epsilon z^{3}\right)$. This implies that $f=k_{4}$.

In view of the fact that $0<\delta<1$, and that $\delta-\beta \geq 0$ provided

$$
1+\frac{\pi^{2}}{16}\left(1-\sqrt{\frac{89}{45}}\right) \geq \rho,
$$

Lemma 3.3 yields

$$
\begin{aligned}
|E| & \leq\left|c_{3}-2 \delta c_{1} c_{2}+\delta c_{1}^{3}\right|+2(\delta-\beta)\left|c_{1} c_{2}\right| \\
& \leq 2+8\left(\frac{32(1-\rho)^{2}}{\pi^{4}}+\frac{4(1-\rho)}{\pi^{2}}-\frac{11}{90}\right) \\
& =2\left(\frac{128(1-\rho)^{2}}{\pi^{4}}+\frac{16(1-\rho)}{\pi^{2}}+\frac{23}{45}\right) .
\end{aligned}
$$

Equality holds if and only if the function $p$ in (3.7) is given by $p(z)=(1+\epsilon z) /(1-\epsilon z)$, that is, $f=k$. This completes the proof.

Theorem 3.5. Let $f(z)=z+a_{2} z^{2}+a_{3} z^{3}+\cdots \in \operatorname{PS}^{*}(\rho)$. For $\mu \in C$ and

$$
\begin{gathered}
\lambda(\mu)=\frac{1}{3}+\frac{16(1-\rho)}{\pi^{2}}(2 \mu-1), \\
\left|a_{3}-\mu a_{2}^{2}\right| \leq \begin{cases}\frac{16(1-\rho)}{3 \pi^{4}}\left|24(1-\rho)(1-2 \mu)+\pi^{2}\right|, & |\lambda(\mu)-1| \geq 1, \\
\frac{8(1-\rho)}{\pi^{2}}, & |\lambda(\mu)-1| \leq 1 .\end{cases}
\end{gathered}
$$

Equality holds in the upper expression of the right inequality if $f=k$ or its rotations, while equality holds in the lower expression of the right inequality if $f=k_{3}$ or its rotations.

Proof. From the relation (3.8), we get

$$
a_{3}-\mu a_{2}^{2}=\frac{4(1-\rho)}{\pi^{2}}\left[c_{2}-\frac{\lambda(\mu)}{2} c_{1}^{2}\right] .
$$

The well-known estimate

$$
\left|c_{2}-\frac{1}{2} c_{1}^{2}\right| \leq 2-\frac{1}{2}\left|c_{1}\right|^{2}
$$


leads to

$$
\left|c_{2}-\frac{\lambda(\mu)}{2} c_{1}^{2}\right| \leq\left|c_{2}-\frac{1}{2} c_{1}^{2}\right|+\frac{|1-\lambda(\mu)|}{2}\left|c_{1}\right|^{2} \leq 2+\frac{|\lambda(\mu)-1|-1}{2}\left|c_{1}\right|^{2},
$$

which yields the desired result.

\section{Convolution properties}

The convolution of $f(z)=\sum_{n=0}^{\infty} a_{n} z^{n}$ and $g(z)=\sum_{n=0}^{\infty} b_{n} z^{n}$ is defined to be the function $(f * g)(z)=\sum_{n=0}^{\infty} a_{n} b_{n} z^{n}$. For $\alpha<1$, denote by $R_{\alpha}$ the class of prestarlike functions of order $\alpha$ consisting of $f \in A$ such that $f *\left((z) /(1-z)^{2-2 \alpha}\right) \in S^{*}(\alpha)$. Here $S^{*}(\alpha)$ is the class of starlike functions of order $\alpha$. An important result in convolution is contained in the following lemma of Ruscheweyh.

Lemma 4.1 [11, page 54]. If $f \in R_{\alpha}, g \in S^{*}(\alpha)$, and $H$ is an analytic function in $U$, then

$$
\frac{f * g H}{f * g}(U) \subset \overline{\mathrm{CO}} H(U)
$$

where $\overline{\mathrm{co}} H(U)$ is the closed convex hull of $H(U)$.

Theorem 4.2. If $f \in R_{\rho}$ and $g \in \operatorname{PS}^{*}(\rho)$, then $f * g \in \operatorname{PS}^{*}(\rho)$.

Proof. Since $g$ also belongs to $S^{*}(\rho)$ and $H(z)=z g^{\prime}(z) / g(z) \prec q_{\rho}(z)$, Lemma 4.1 yields

$$
\frac{z(f * g)^{\prime}}{f * g}(U)=\frac{f * z g^{\prime}}{f * g}(U)=\frac{f * g\left(z g^{\prime} / g\right)}{f * g}(U) \subset \overline{\mathrm{co}} \frac{z g^{\prime}}{g}(U) \subset \bar{\Omega}_{\rho},
$$

and hence, $f * g \in \operatorname{PS}^{*}(\rho)$.

Since $R_{1 / 2}=S^{*}(1 / 2)$ (see [11]), and $R_{0}=C$, where $C$ is the class of convex functions in $A$, a similar proof also yields the following result.

Corollary 4.3. (a) If $f, g \in \mathrm{PS}^{*}(\rho)$ for $\rho \geq 1 / 2$, then $f * g \in \operatorname{PS}^{*}(\rho)$.

(b) If $f \in C$ and $g \in \operatorname{PS}^{*}(\rho)$, then $f * g \in \operatorname{PS}^{*}(\rho)$.

\section{Acknowledgments}

This research was supported by a Universiti Sains Malaysia Fundamental Research Grant. The author is greatly indebted to Professor V. Ravichandran for his helpful comments in the preparation of this paper.

\section{References}

[1] R. Aghalary and S. R. Kulkarni, Certain properties of parabolic starlike and convex functions of order $\rho$, Bull. Malays. Math. Sci. Soc. (2) 26 (2003), no. 2, 153-162.

[2] R. M. Ali, Coefficients of the inverse of strongly starlike functions, Bull. Malays. Math. Sci. Soc. (2) 26 (2003), no. 1, 63-71.

[3] R. M. Ali and V. Singh, Coefficients of parabolic starlike functions of order $\rho$, Computational Methods and Function Theory 1994 (Penang), Ser. Approx. Decompos., vol. 5, World Scientific Publishing, New Jersey, 1995, pp. 23-36. 
[4] A. W. Goodman, On uniformly convex functions, Ann. Polon. Math. 56 (1991), no. 1, 87-92.

[5] S. K. Lee, Characterizations of parabolic starlike functions and the generalized uniformly convex functions, Master's thesis, Universiti Sains Malaysia, Penang, Malaysia, 2000.

[6] W. C. Ma and D. Minda, Uniformly convex functions, Ann. Polon. Math. 57 (1992), no. 2, 165175.

[7] _ Uniformly convex functions. II, Ann. Polon. Math. 58 (1993), no. 3, 275-285.

[8] _ A unified treatment of some special classes of univalent functions, Proceedings of the Conference on Complex Analysis (Tianjin, 1992), Conf. Proc. Lecture Notes Anal., I, International Press, Massachusetts, 1994, pp. 157-169.

[9] F. Rønning, On starlike functions associated with parabolic regions, Ann. Univ. Mariae CurieSkłodowska Sect. A 45 (1991), 117-122.

[10] Uniformly convex functions and a corresponding class of starlike functions, Proc. Amer. Math. Soc. 118 (1993), no. 1, 189-196.

[11] S. Ruscheweyh, Convolutions in Geometric Function Theory, Séminaire de Mathématiques Supérieures, vol. 83, Presses de l'Université de Montréal, Quebec, 1982.

[12] T. N. Shanmugam and V. Ravichandran, Certain properties of uniformly convex functions, Computational Methods and Function Theory 1994 (Penang), Ser. Approx. Decompos., vol. 5, World Scientific Publishing, New Jersey, 1995, pp. 319-324.

Rosihan M. Ali: School of Mathematical Sciences, Universiti Sains Malaysia, 11800 Penang, Malaysia

E-mail address: rosihan@cs.usm.my 


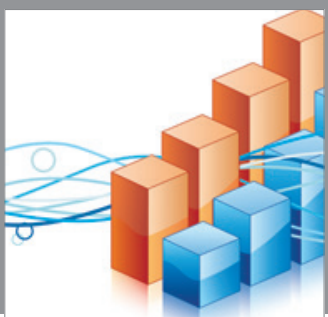

Advances in

Operations Research

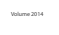

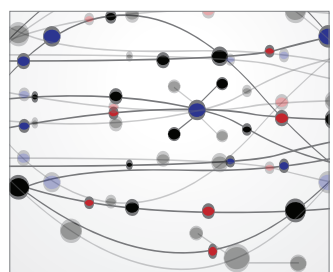

\section{The Scientific} World Journal
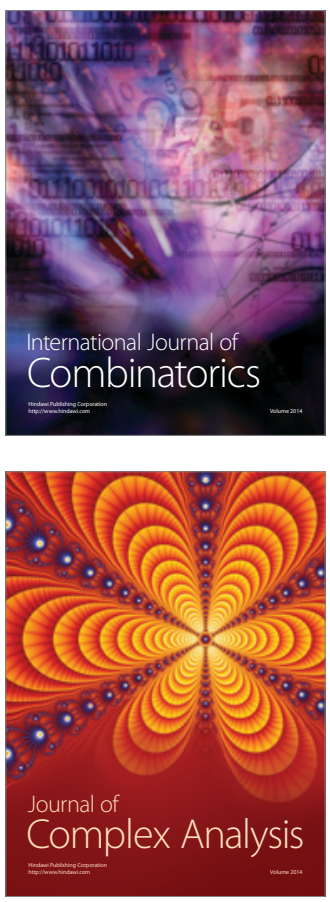

International Journal of

Mathematics and

Mathematical

Sciences
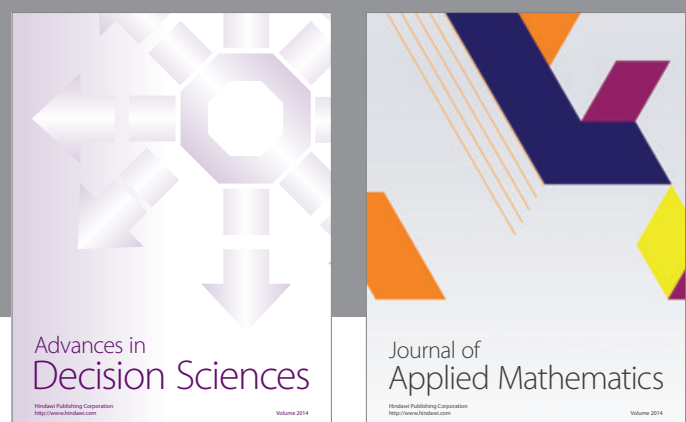

Journal of

Applied Mathematics
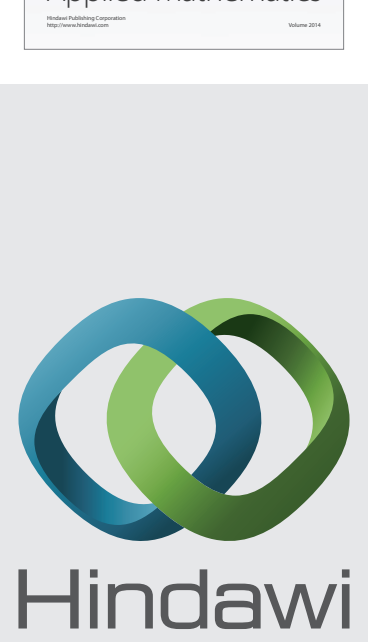

Submit your manuscripts at http://www.hindawi.com
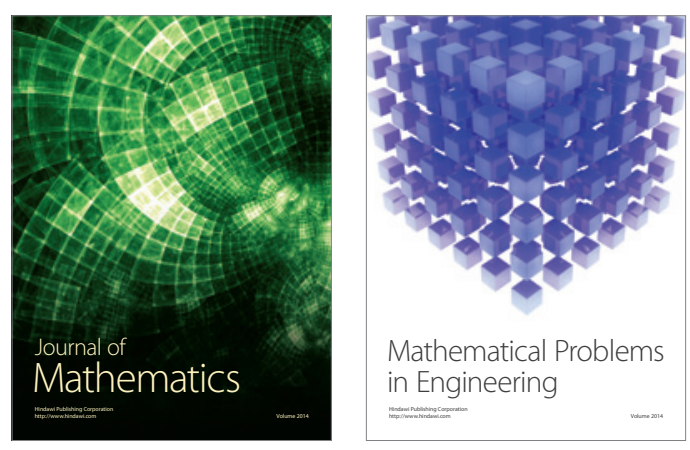

Mathematical Problems in Engineering
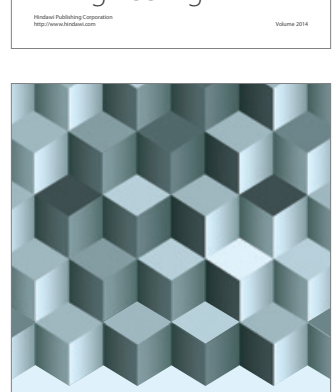

Journal of

Function Spaces
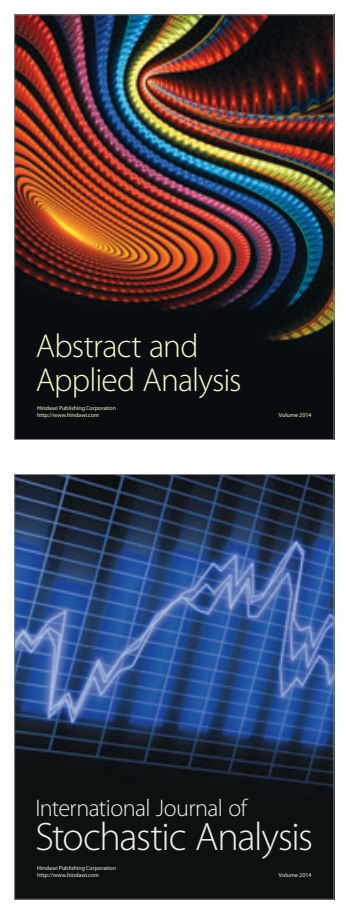

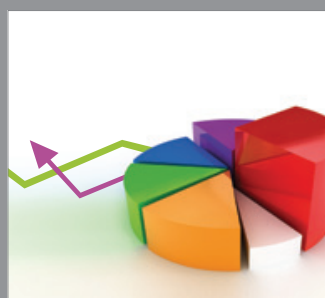

ournal of

Probability and Statistics

Promensencen
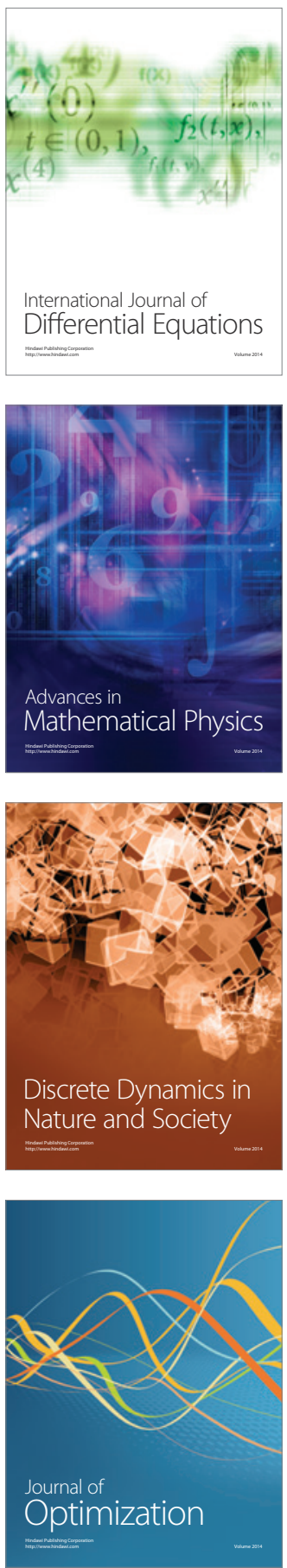